\title{
Poli- en isomorfisme in halogeengesubstitueerde 4-(fenielamino)pent-3-en-2-oon verbindings
}

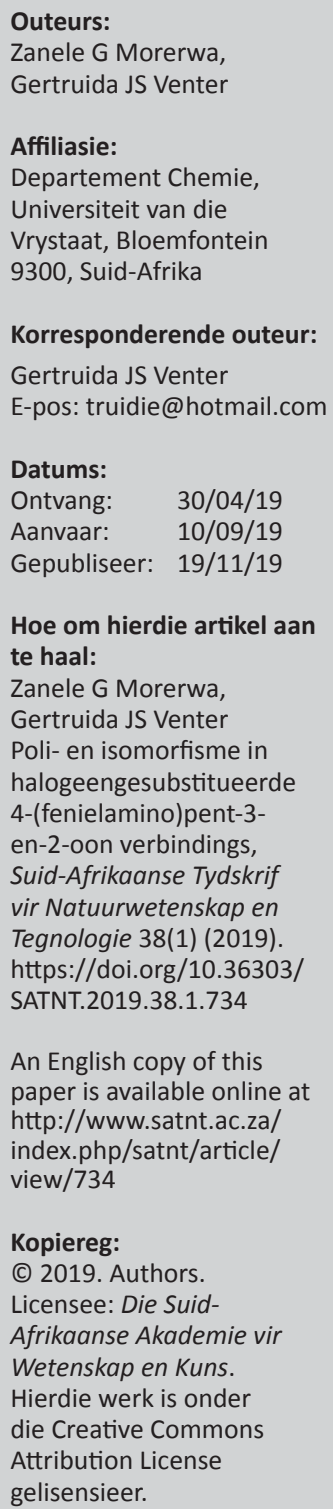

'n Reeks halogeengesubstitueerde feniel- $\beta$-enaminoketonaat verbindings (N,Oskenkeratome) word voorgelê. Die invloed van halogeensubstitusie aan die fenielring op vastetoestand parameters is ondersoek. X-straal strukture van 4-(4-fluorofenielamino) pent-3-en-2-oon (4-F-PhonyH), 4-(4-chlorofenielamino)pent-3-en-2-oon (4-Cl-PhonyH), en 4-(4-bromofeniel-amino)pent-3-en-2-oon (4-Br-PhonyH) is gerapporteer. Alhoewel 4-F-PhonyH en die chloro- en bromo-eweknieë struktureel soortgelyk is, is daar daadwerklike verskille in terme van dihedriese hoeke en intermolekulêre interaksies. Al drie verbindinge vertoon polimorfisme in twee verskillende kristalstelsels, met 4-F-PhonyH wat in $P 2_{1} / c$, beide 4-Cl-PhonyH en 4-Br-PhonyH in $C_{c}$, en al drie in $P \overline{1}$ kristalliseer.

Sleutelwoorde: Enaminoketoon, polimorfisme, isomorfisme.

Poli- and isomorphism in halogen-substituted 4-(phenylamino)pent-3-en-2-one compounds: A range of halogen-substituted phenyl $\beta$-enaminoketonato compounds $(\mathrm{N}, \mathrm{O}-$ donor atoms) as ligand models is presented. The influence of halogen substitution on the phenyl ring on solid state parameters was investigated by $X$-ray structures of 4-(4-fluorophenylamino)pent-3-en-2-one (4-F-PhonyH), 4-(4-chlorophenylamino)pent-3en-2-one (4-Cl-PhonyH), and 4-(4-bromophenyl-amino)pent-3-en-2-one (4-Br-PhonyH), which are reported. Although structurally very similar, 4-F-PhonyH and its chloro- and bromo-substituted counterparts differ significantly with regard to dihedral angles and intermolecular interactions. All three compounds show polymorphism in two different crystal systems, with 4 -F-PhonyH crystallising in $\mathrm{P} 2{ }_{1} / \mathrm{c}$, both $4-\mathrm{Cl}-\mathrm{PhonyH}$ and $4-\mathrm{Br}-$ PhonyH crystallising in $C c$, and all three crystallising in $P \overline{1}$.

Keywords: enaminoketone, polymorphism, isomorphism

\section{Inleiding}

'n Welbekende ligandsisteem in organiese chemie is die $\beta$-diketoonverbinding AcacH (asetielasetoon). Menige derivate hiervan is tot op datum vervaardig (Groom, et al., 2016), en sluit stikstofgesubstitueerde enaminoketone, ook bekend as ketoïmiene, in. Elektronryke enaminoketone bevat stikstof- en suurstofatome asook 'n alkeen, en het toepassingsmoontlikhede in verskeie velde, insluitende vloeistofkristalle (Pyżuk, et al., 1993), fluorosensiestudies (Xia, et al., 2008), katalise (Nair, et al., 2002) en medisyne (Tan, et al., 2008; Chen \& Rhodes, 1996). Al verskil enaminoketone in ontwerp, reageer hulle oor die algemeen as mono-anioniese, bidentate ligande. Halogene is alomteenwoordig in beide anorganiese en organiese chemie en kom dikwels as monodentate bruggingsligande of substituente in organiese verbindings voor. Die steriese impak van halogene het potensiële toepassings in supramolekulêre chemie en kristalingenieurswese waar die halogeenatome direk betrokke is by die vorming van intermolekulêre interaksies (Fox, et al., 2004; Csöregh, et al., 2001).

Hierdie ondersoek fokus op halogeengesubstitueerde 4-(fenielamino)pent-3-en-2-oon (PhonyH, Shaheen, et al., 2006; Figuur 1) tipe verbindings en die invloed van verskillende halogene op dieselfde posisie op die fenielring, op die geometrie en kristalpakking, soos waargeneem uit vastetoestand studies.

Die duidelike verskille in die Van der Waals radiusse van halogene $(1.47 \AA$ vir $\mathrm{F}, 1.75 \AA$ vir $\mathrm{Cl}$ en $1.83 \AA$ vir Br; Bondi, 1964; Mantina, et al., 1987) skep die vraag van watter impak die steriese eienskappe van die substituente op die fenielring op die geometriese parameters van isostrukturele verbindings en hulle onderskeie pakkingsmodusse het. Hierdie navraag is van kardinale belang in die ontwerp van komplekse wat hierdie verbindings as ligandstelsel gebruik. 


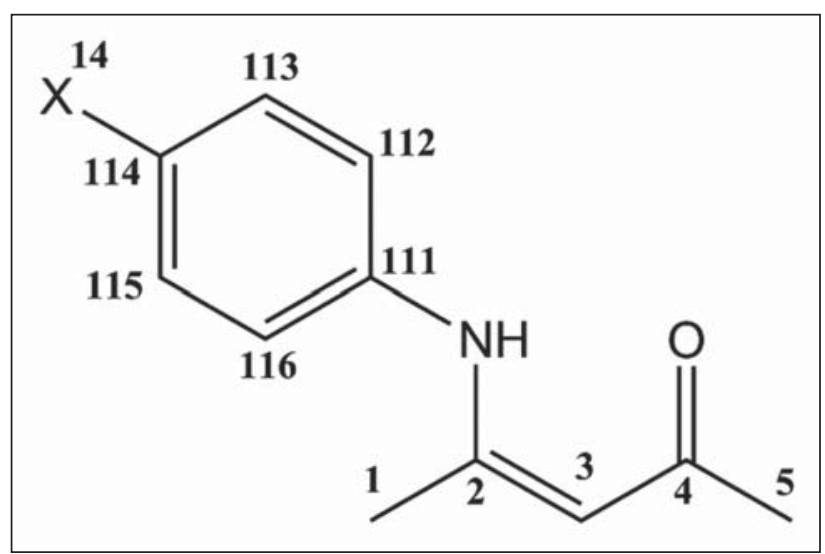

FIGUUR 1: Skematiese voorstelling van 4-(X-fenielamino)pent-3-en-2-oon (4-X-PhonyH, waar $\mathrm{X}=\mathrm{F}, \mathrm{Cl}, \mathrm{Br}$ ) wat die benamingsmetode wat deurgaans in die artikel gebruik word, illustreer. Vir die C-atome op die fenielring dui die eerste getal op die molekuulnommer, die tweede getal dui op ringnommer en die derde getal dui die posisie op die ring aan. Vir halogeensubstitusies op die fenielring, aangedui deur $\mathrm{X}$, dui die eerste getal op die molekuulnommer terwyl die tweede getal die posisie op die fenielring aandui.

Die bespreking dek die polimorwe van 4-(4-fluorofenielamino)pent-3-en-2-oon (4-F-PhonyH, I en II), 4-(4-chlorofenielamino)pent-3-en-2-one (4-Cl-PhonyH, III en IV) asook 4-(4-bromofenielamino)pent-3-en-2-one (4-BrPhonyH, V en VI) en sluit die ondersoek van die elektroniese invloed op die geometriese parameters en pakkingstyle in. Polimorwe van die strukture vir 4-Cl-PhonyH en $4-\mathrm{Br}-$ PhonyH is voorheen gerapporteer (Olejník, et al., 2012; Sergienko, et al., 1987) en is in die Cambridge Crystal Structure Database (CSD) opgeneem onder die kodes WUQDOD en GEZSEJ. Ander gepubliseerde strukture soortgelyk aan PhonyH sluit metiel- (Venter, et al., 2010a; 2010b; 2012a; 2014), fluoro- (Gordon, et al., 2002), chloro(Venter, et al., 2012b; 2013), bromo- (Venter, et al., 2011), hidroksiel- (Parekh, et al., 2007) en nitrobevattende (Da Silva, et al., 1993) verbindings in.

\section{Materiaal en Metodes}

\section{Sintese}

Alle reagense is gebruik soos aangekoop. Die sintese van 4-Cl-PhonyH word gerapporteer as voorbeeld van 'n tipiese voorbereidingsmetode.

'n Oplossing van asetielasetoon (11.07 g, $0.1106 \mathrm{~mol}$ ), 4-chloro-anilien (12.77 g, $0.1001 \mathrm{~mol})$ en 2 druppels $\mathrm{H}_{2} \mathrm{SO}_{4}$ (kons.) in $150 \mathrm{ml}$ benseen is vir 24 uur in ' $\mathrm{n}$ DeanStark-val gerefluks, gefiltreer en toegelaat om te kristalliseer. Kristalle geskik vir X-straaldiffraksie is verkry (17.14 g, $81.67 \%$ opbrengs). Alle N,O-bidentaatverbindings is deur middel van dieselfde prosedure voorberei. Hierdie produkte is stabiel in lug en lig oor 'n tydperk van verskeie jare.

Die KMR data vir 4-Cl-PhonyH word voorsien as voorbeeld vir hierdie stelsel; die volle spektra is beskikbaar in die bylaag: ${ }^{1} \mathrm{H}$ NMR (300.13 MHz, $\left.\mathrm{C}_{6} \mathrm{D}_{6}, 25^{\circ} \mathrm{C}\right)$ : $1.44(\mathrm{~s}, 5 \mathrm{H})$, 2.00 (s, 1H), 4.94 (s, 3H), 6.39 (m, 113H; 115H), 6.87 (d, 112H; 116H). ${ }^{13} \mathrm{C} \mathrm{NMR}\left(75.48 \mathrm{MHz}, \mathrm{C}_{6} \mathrm{D}_{6}, 25^{\circ} \mathrm{C}\right): 19.3$ (s, 1C), 29.22 (s, 5C), 98.44 (s, 3C), 125.43 (s, 113C; 115C), 129.19 (s, 112C; 116C), 129.82 (s, 111C), 130.37 (s, 114C), 158.57 (s, 2C), 195.99 (s, 4C).

\section{Enkelkristal X-straalkristallografie}

Die data-opname is gedoen op ' $n$ Bruker Apex II $4 \mathrm{~K}$ CCD diffraktometer met Mo $K \alpha(0.71073 \AA)$ en $\phi$ en $\omega$ skanderings by $100(2) \mathrm{K}$. Alle refleksies is saamgevoeg en geïntegreer met SAINT-PLUS (Bruker, 2004) en is aangepas vir Lorentz, polariserings- en absorpsie-effekte deur SADABS (Bruker, 1998). Die strukture is opgelos deur direkte metodes en verfyn deur vol-matriks kleinste kwadraatsiklusse deur SHELX-97 (Sheldrick, 2008) en as deel van die WinGX (Farrugia, 2012) pakket met $\Sigma\left(|| F_{o}|-| F_{c}||\right)^{2}$ geminimaliseer. Alle nie-H-atome is verfyn met anisotropiese verplasingsparameters, terwyl $\mathrm{H}$-atome beperk is tot moederatoomliggings deur middel van 'n rymodel (aromatiese $\mathrm{C}-\mathrm{H}=0.95 \AA$; alifatiese $\mathrm{C}-\mathrm{H}=0.98 \AA$ ). Die figure is verkry met die DIAMOND (Putz \& Brandenburg, 2018) Visual Crystal Structure Information System sagteware. Kristallografiese data vir die strukture is beskikbaar op die 'Cambridge Structural Database' onder CCDC nommers1910214-1910217.

\section{Resultate}

Die uitgangstowwe reageer maklik om die teiken $\mathrm{N}, \mathrm{O}-$ bidentate verbindings $\overline{P 1} 4$-F-PhonyH (I), $P 2_{1} / c$ 4-F-PhonyH (II), Cc 4-Cl-PhonyH (III), en Cc 4-Br-PhonyH (V) te vorm wat in Figuur 2 aangedui word. Die $P \overline{1}$ polimorwe van 4-Cl-PhonyH (IV) en 4-Br-PhonyH (VI) word volledigheidshalwe ingesluit.

Verbinding I kristalliseer met twee onafhanklike molekules in die asimmetriese eenheid in $P \overline{1}$, terwyl verbindings III en IV met vier in $C c$ kristalliseer. Alle ander polimorwe kristalliseer met slegs een. Alle verbindings is stabiel onder omgewingstoestande. Tabel 1 bevat die basiese datainsameling en verfyningsparameters van die kristalstrukture wat hierbo genoem is. Belangrike bindingsafstande en -hoeke vir elke stel polimorwe word in Tabelle 2 tot 4 aangedui. 


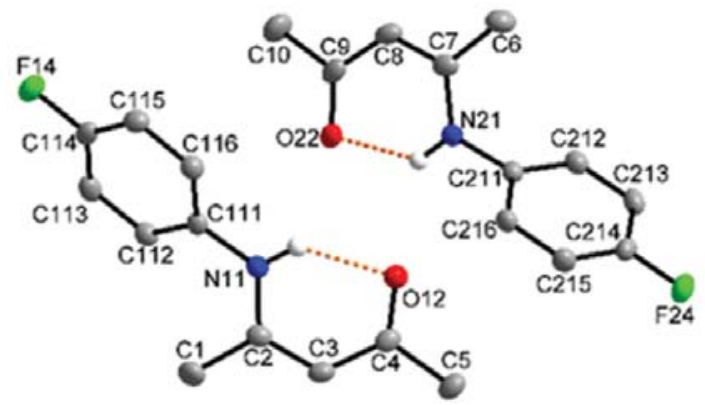

I

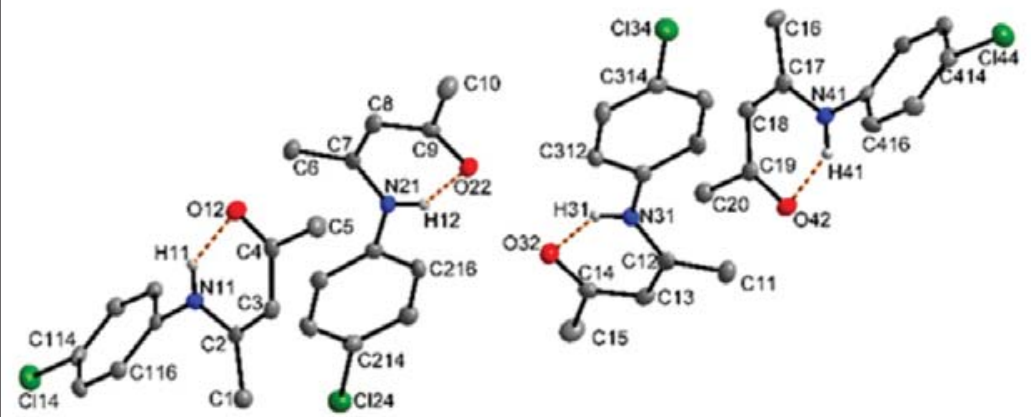

III
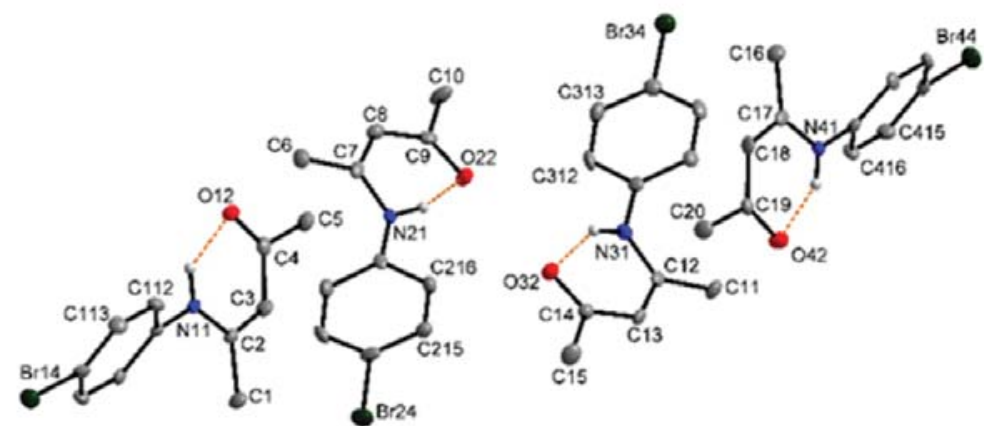

V

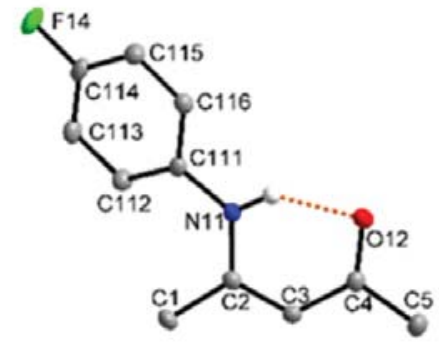

II

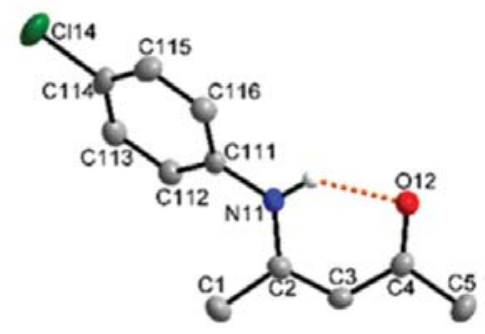

IV

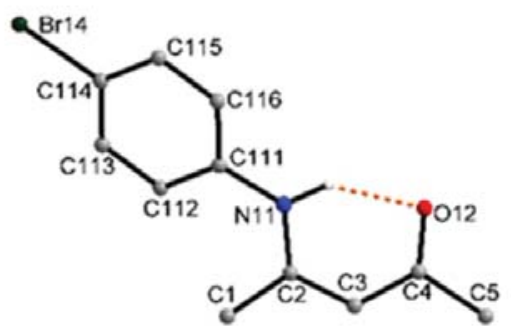

VI

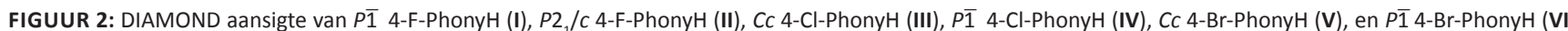
( $50 \%$ waarskynlikheid verplasingsellipsoïede), waar PhonyH =4-(fenielamino)pent-3-en-2-oon. Minderbelangrike waterstofatome is uitgelaat vir duidelikheid. Vir die $\mathrm{C}$-atome in die fenielring dui die eerste syfer die molekuulnommer, die tweede die ringnommer, en die derde die posisie van die atoom op die ring aan. Sommige atoomnommers is uitgelaat ter wille van duidelikheid maar alle ringe is op dieselfde konsekwente manier gemerk. Vir die halogeenatome dui die eerste syfer die molekuulnommer en die tweede syfer die posisie van die atoom op die ring aan. 
TABEL 1: Strukturele inligting van die halogeenderivate van PhonyH.

\begin{tabular}{|c|c|c|c|c|c|c|}
\hline Verbinding & $\begin{array}{l}\text { 4-F-PhonyH } \\
\text { (I) }\end{array}$ & $\begin{array}{l}\text { 4-F-PhonyH polimorf } \\
\text { (II) }\end{array}$ & $\begin{array}{l}\text { 4-Cl-PhonyH } \\
\text { (III) }\end{array}$ & $\begin{array}{l}\text { 4-Cl-PhonyH } \\
\text { polimorf (IV) }^{\text {a }}\end{array}$ & $\begin{array}{c}\text { 4-Br-PhonyH } \\
\text { (V) }\end{array}$ & $\begin{array}{c}\text { 4-Br-PhonyH polimorf } \\
\text { (VI) }\end{array}$ \\
\hline Kristal kleur & Kleurloos & Kleurloos & Geel & Kleurloos & Geel & Kleurloos \\
\hline Kristalstelsel & Triklinies & Monoklinies & Monoklinies & Triklinies & Monoklinies & Triklinies \\
\hline Ruimtegroep & $P \overline{1}$ & $P 2_{1} / c$ & $C c$ & $P \overline{1}$ & $C c$ & $P \overline{1}$ \\
\hline Temperatuur (K) & $100(2)$ & $100(2)$ & $100(2)$ & $150(1)$ & $100(2)$ & 295 \\
\hline$a(\AA ̊)$ & $8.9561(2)$ & $9.2410(8)$ & $14.1370(7)$ & $6.9730(5)$ & $14.2020(2)$ & $6.977(2)$ \\
\hline$b(\AA ̊)$ & $10.8218(2)$ & $10.8140(9)$ & $14.5370(6)$ & $7.9481(4)$ & $14.6910(2)$ & $8.154(2)$ \\
\hline$c(\AA ̊)$ & $12.3640(3)$ & 10.1670(9) & $20.4780(8)$ & $10.5520(4)$ & $20.8920(3)$ & $10.821(3)$ \\
\hline$\alpha\left({ }^{\circ}\right)$ & $111.429(1)$ & 90 & 90 & $99.767(4)$ & 90 & $100.11(2)$ \\
\hline$\beta\left(^{\circ}\right)$ & $97.691(1)$ & $107.468(4)$ & $100.516(1)$ & $100.433(5)$ & $101.244(1)$ & $100.73(2)$ \\
\hline$\gamma\left({ }^{\circ}\right)$ & $108.876(1)$ & 90 & 90 & $109.868(4)$ & 90 & $108.67(2)$ \\
\hline$V(\AA ̊ 3)$ & $1011.37(4)$ & $969.2(2)$ & $4137.7(3)$ & $523.74(5)$ & $4275.3(1)$ & 554.435 \\
\hline$Z$ & 4 & 4 & 16 & 2 & 16 & 2 \\
\hline$D_{\text {calc }}\left(\mathrm{g} \cdot \mathrm{cm}^{-3}\right)$ & 1.269 & 1.3242 & 1.3462 & 1.33 & 1.5792 & 1.418 \\
\hline Kristalgrootte $\left(\mathrm{mm}^{3}\right)$ & $0.35 \times 0.29 \times 0.24$ & $0.71 \times 0.56 \times 0.28$ & $0.17 \times 0.13 \times 0.1$ & $0.32 \times 0.19 \times 0.17$ & $0.50 \times 0.48 \times 0.45$ & \\
\hline$\mu\left(\mathrm{mm}^{-1}\right)$ & 0.094 & 0.098 & 0.334 & 0.33 & 3.812 & \\
\hline Refleksies gemeet & 11052 & 36822 & 23579 & 10122 & 24956 & \\
\hline uniek, $\mathrm{R}_{\mathrm{int}}$ & $4405,0.0222$ & $2418,0.0507$ & $8986,0.0311$ & $2265,0.0794$ & $9849,0.0513$ & \\
\hline Getal parameters & 265 & 133 & 513 & 135 & 513 & \\
\hline$R 1\left[I^{3} 2 \sigma(I)\right]$ & 0.0384 & 0.0406 & 0.0335 & 0.0433 & 0.0357 & \\
\hline$w R_{2}$ (alle data, $F^{2}$ ) & 0.1081 & 0.1337 & 0.0785 & 0.1133 & 0.0712 & \\
\hline GOF on $F^{2}$ & 1.053 & 1.061 & 1.038 & 1.075 & 0.936 & \\
\hline$\Delta \rho \mathrm{min} / \mathrm{maks}(\mathrm{e} . \AA \AA-3)$ & $0.23 /-0.21$ & $0.37 /-0.43$ & $0.25 /-0.29$ & $0.275 /-0.391$ & $0.51 /-0.80$ & \\
\hline
\end{tabular}

${ }^{a}$ Aangepas vanuit Olejnik et al., 1987. Kopiereg: $\underline{\underline{I U C r}}$, met toestemming. ${ }^{b}$ Aangepas vanuit Sergienko et al., 1987. Kopiereg: $\underline{\mathrm{IUCr}}, \mathrm{met}$ toestemming.

TABEL 2: Uitgesoekte bindingsafstande $(\AA ̊)$ en -hoeke $\left({ }^{\circ}\right)$ in 4-F-PhonyH polimorwe.

\begin{tabular}{l|cc|c}
\hline \multirow{2}{*}{} & \multicolumn{2}{|c|}{$\mathbf{I}$} & \multirow{2}{*}{ II } \\
\cline { 2 - 3 } & $1.424(2)$ & $1.424(2)$ & $1.412(2)$ \\
\hline $\mathrm{N}_{11}-\mathrm{C}_{111}$ & $1.340(2)$ & $1.347(2)$ & $1.354(1)$ \\
$\mathrm{N}_{11}-\mathrm{C}_{2}$ & $1.247(2)$ & $1.247(2)$ & $1.254(1)$ \\
$\mathrm{O}_{12}-\mathrm{C}_{4}$ & $2.668(1)$ & $2.709(2)$ & $2.641(1)$ \\
$\mathrm{N}_{11} \ldots \mathrm{O}_{12}$ & $1.382(2)$ & $1.375(2)$ & $1.383(2)$ \\
$\mathrm{C}_{2}-\mathrm{C}_{3}$ & $1.426(2)$ & $1.427(2)$ & $1.428(2)$ \\
$\mathrm{C}_{3}-\mathrm{C}_{4}$ & $136(2)$ & $134(2)$ & $142(2)$ \\
$\mathrm{N}_{11}-\mathrm{H}_{11} \ldots \mathrm{O}_{12}$ & $-0.5(1)$ & $-1.0(1)$ & $1.72(9)$ \\
$\mathrm{N}_{11}-\mathrm{C}_{2}-\mathrm{C}_{4}-\mathrm{O}_{12}$ & $58.99(4)$ & $31.67(6)$ & $31.97(4)$ \\
Dihedriese hoek $^{c}$ & &
\end{tabular}

c Gedefinieer as die torsiehoek tussen die N-C-C-C-O vlak en die fenielring. 'n Positiewe hoek dui op'n kloksgewyse rotasie.

TABEL 3: Uitgesoekte bindingsafstande $(\AA ̊)$ en -hoeke $\left({ }^{\circ}\right)$ in 4-Cl-PhonyH polimorwe.

\begin{tabular}{|c|c|c|c|c|c|}
\hline & \multicolumn{4}{|c|}{ III } & \multirow[t]{2}{*}{ IV } \\
\hline & 1 & 2 & 3 & 4 & \\
\hline $\mathrm{N}_{11}-\mathrm{C}_{111}$ & $1.423(3)$ & $1.419(3)$ & $1.410(3)$ & $1.419(3)$ & $1.417(2)$ \\
\hline $\mathrm{N}_{11}-\mathrm{C}_{2}$ & $1.352(3)$ & $1.344(3)$ & $1.350(3)$ & $1.353(3)$ & $1.348(2)$ \\
\hline $\mathrm{O}_{12}-\mathrm{C}_{4}$ & $1.247(3)$ & $1.252(3)$ & $1.250(3)$ & $1.246(3)$ & $1.243(2)$ \\
\hline $\mathrm{N}_{11} \ldots \mathrm{O}_{12}$ & $2.676(3)$ & $2.620(3)$ & $2.634(3)$ & $2.678(3)$ & $2.705(2)$ \\
\hline $\mathrm{C}_{2}-\mathrm{C}_{3}$ & $1.378(3)$ & $1.378(3)$ & $1.380(3)$ & $1.376(3)$ & $1.375(3)$ \\
\hline $\mathrm{C}_{3}-\mathrm{C}_{4}$ & $1.427(3)$ & $1.422(3)$ & $1.421(3)$ & $1.434(3)$ & $1.434(3)$ \\
\hline $\mathrm{N}_{11}-\mathrm{H}_{11} \ldots \mathrm{O}_{12}$ & $134.8(1)$ & $137.5(2)$ & $139.4(2)$ & $134.7(2)$ & $137(2)$ \\
\hline $\mathrm{N}_{11}-\mathrm{C}_{2}-\mathrm{C}_{4}-\mathrm{O}_{12}$ & $1.5(2)$ & $1.7(2)$ & $-3.0(2)$ & $-1.7(2)$ & $0.7(1)$ \\
\hline Dihedriese hoek $^{c}$ & $51.71(8)$ & $-41.30(8)$ & $38.36(9)$ & $-52.71(7)$ & $45.45(5)$ \\
\hline
\end{tabular}

'Gedefinieer as die torsiehoek tussen die N-C-C-C-O vlak en die fenielring. 'n Positiewe hoek dui op 'n kloksgewyse rotasie. 
TABEL 4: Uitgesoekte bindingsafstande $(\AA ̊)$ en -hoeke $\left({ }^{\circ}\right)$ in 4-Br-PhonyH polimorwe.

\begin{tabular}{|c|c|c|c|c|c|}
\hline & \multicolumn{4}{|c|}{ V } & \multirow[t]{2}{*}{ VI } \\
\hline & 1 & 2 & 3 & 4 & \\
\hline $\mathrm{N}_{11}-\mathrm{C}_{111}$ & $1.430(4)$ & $1.392(4)$ & $1.417(4)$ & $1.413(4)$ & $1.44(1)$ \\
\hline $\mathrm{N}_{11}-\mathrm{C}_{2}$ & $1.360(4)$ & $1.341(4)$ & $1.359(4)$ & $1.370(4)$ & $1.34(1)$ \\
\hline $\mathrm{O}_{12}-\mathrm{C}_{4}$ & $1.244(4)$ & $1.251(4)$ & $1.252(4)$ & $1.247(4)$ & $1.25(1)$ \\
\hline $\mathrm{N}_{11} \ldots \mathrm{O}_{12}$ & $2.675(4)$ & $2.635(4)$ & $2.629(4)$ & $2.664(4)$ & $2.70(1)$ \\
\hline $\mathrm{C}_{2}-\mathrm{C}_{3}$ & $1.368(5)$ & $1.372(5)$ & $1.379(5)$ & $1.373(4)$ & $1.37(1)$ \\
\hline $\mathrm{C}_{3}-\mathrm{C}_{4}$ & $1.431(5)$ & $1.432(5)$ & $1.430(4)$ & $1.427(5)$ & $1.41(1)$ \\
\hline $\mathrm{N}_{11}-\mathrm{H}_{11} \ldots \mathrm{O}_{12}$ & $131.5(1)$ & $152.9(2)$ & $145.5(2)$ & $139.3(2)$ & $139(6)$ \\
\hline $\mathrm{N}_{11}-\mathrm{C}_{2}-\mathrm{C}_{4}-\mathrm{O}_{12}$ & $-1.8(3)$ & $-3.4(3)$ & $2.3(3)$ & $1.0(3)$ & $0.7(7)$ \\
\hline Dihedriese hoek $^{c}$ & $51.54(8)$ & $-37.0(1)$ & $39.5(1)$ & $-51.61(9)$ & $47.9(3)$ \\
\hline
\end{tabular}

${ }^{c}$ Gedefinieer as die torsiehoek tussen die N-C-C-C-O vlak en die fenielring. 'n Positiewe hoek dui op 'n kloksgewyse rotasie.

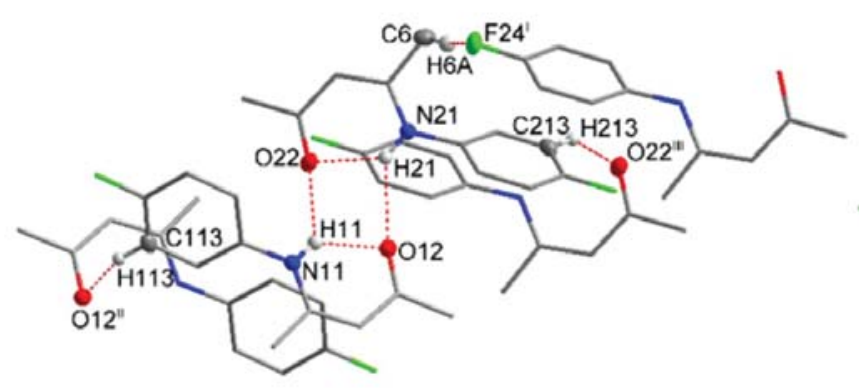

I

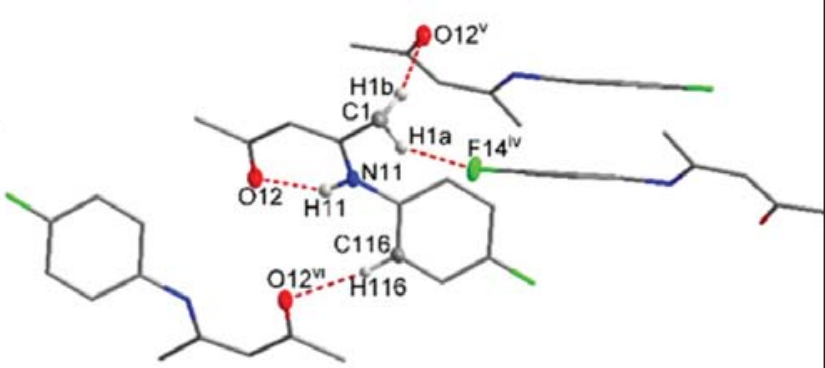

II
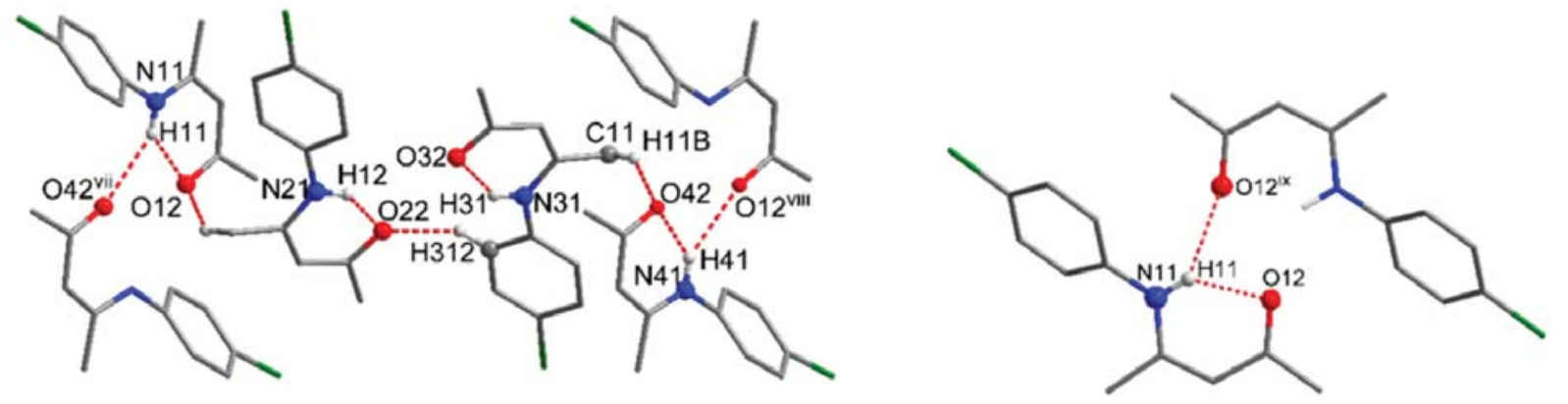

III

IV
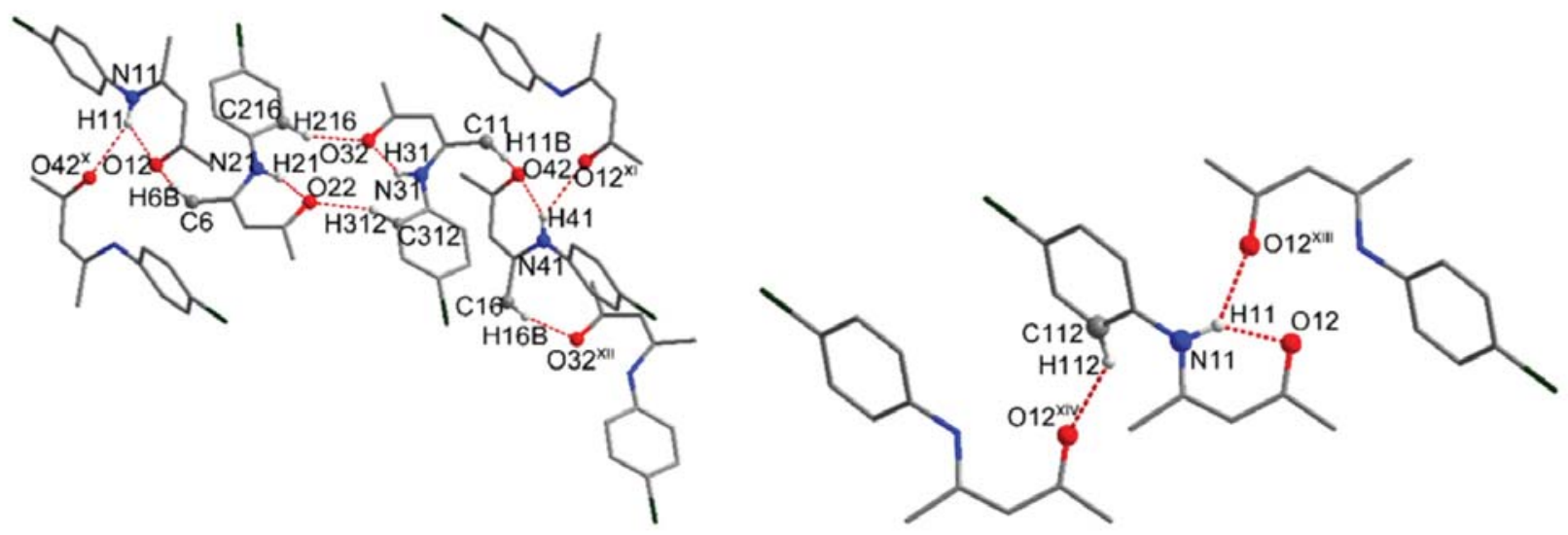

VI

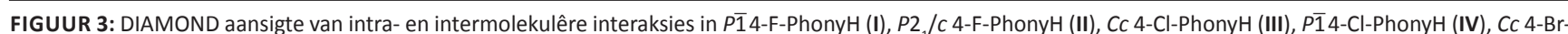
PhonyH (V), en $P \overline{1}$ 4-Br-PhonyH (VI), waar PhonyH = 4-(fenielamino)pent-3-en-2-oon. Minderbelangrike waterstofatome is uitgelaat vir duidelikheid. 
Al die verbindings onder bespreking vertoon intramolekulêre $\mathrm{N}-\mathrm{H} \cdots \mathrm{O}$ interaksies, terwyl intermolekulêre $\mathrm{N}-\mathrm{H} \cdots \mathrm{O}$ en $\mathrm{C}-\mathrm{H} \cdots \mathrm{O}$ interaksies ook algemeen is. Die interaksies word in Figuur 3 geilllustreer met die parameters in Tabel A.1 in die bylaag opgesom.

\section{Bespreking}

4-X-PhonyH derivate toon ' $\mathrm{n}$ duidelike tendens om in veelvuldige kristalstelsels en ruimtegroepe te kristalliseer soos aangedui in Tabel 1. Die moontlikheid dat temperatuur 'n rol hierin kan speel soos aangedui deur die verskillende vir 4-Cl-PhonyH en 4-Br-PhonyH in literatuur, word weerlê deur die feit dat polimorwe van 4-F-PhonyH waargeneem word ten spyte van identiese temperature. In alle gevalle vertoon 4-F-PhonyH duidelike verskille in vergelyking met die $\mathrm{Cl}$ en $\mathrm{Br}$ eweknieë, en al kristalliseer alle verbindings onder bespreking in een geval in $P \overline{1}$, verskil die eenheidselparameters aansienlik met 4-Cl-PhonyH en 4-Br-PhonyH, terwyl dié van 4-Cl-PhonyH en 4-BrPhonyH nader aan mekaar is. Alhoewel die radius van $\mathrm{F}$ aansienlik kleiner is as dié van $\mathrm{Cl}$ en $\mathrm{Br}$, vergroot die twee 4-F-PhonyH molekule in die asimmetriese eenheid die eenheidselparameters tot so 'n mate dat die eenheidselvolume verdubbel. Al die strukture wat in $P \overline{1}$ kristalliseer deel een eienskap, naamlik intra- en intermolekulêre $\mathrm{N}-\mathrm{H} \cdots \mathrm{O}$ bindings met gevurkte $\mathrm{H}_{11}$ (of $\mathrm{H}_{21}$, waar teenwoordig) waterstofbindings wat as kristallografiese dimerisme manifesteer. Aangesien waterstofbindings langafstand interaksies is, kan meer as een ontvangerentiteit $\mathrm{A}$ aan 'n skenkergroep D-H bind. Wanneer daar twee ontvangers $A_{1}$ en $A_{2}$ is, word die interaksie as 'n gevurkte waterstofbinding $\mathrm{D}-\mathrm{H} \cdots\left(\mathrm{A}_{1}, \mathrm{~A}_{2}\right)$ (Francl, et al., 1982) geklassifiseer, soos wat in hierdie verbindings waargeneem is. Hierdie dimeriese rangskikking is nie in die $P 2_{1} / c$ polimorf van 4 -F-PhonyH teenwoordig nie, maar kom weer voor in die $\mathrm{Cc}$ polimorwe van 4-Cl-PhonyH en 4-BrPhonyH. Kristallografiese polimerisme deur III en $\mathbf{V}$ vertoon is duidelik sigbaar in Figuur 3, met gevurkte waterstofbindings teenwoordig vir $\mathrm{H}_{11}$ en $\mathrm{H}_{41}$. Hierdie tipe polimerisme word moontlik gemaak deur die intermolekulêre $\mathrm{N}-\mathrm{H} \cdots \mathrm{O}$ interaksies tussen aangrensende molekule.

Die grootste verskil tussen die geometriese parameters van die polimorwe van 4-F-PhonyH, verbindings I en II, is in die $\mathrm{N}_{11} \ldots \mathrm{O}_{12}$ afstande, naamlik 2.668(1) Å en 2.709(2) $\AA$ vir I en 2.641(1) ^ vir II, onderskeidelik. Dit dui op 'n vergroting in die 'byt' van die ligand vir polimorf $\mathbf{I}$, maar is teenstrydig met die $\mathrm{N}_{11}-\mathrm{H}_{11} \ldots \mathrm{O}_{12}$ afstande van $136(2)^{\circ}$ en $134(2)^{\circ}$ vir I en $142(1)^{\circ}$ vir II en die $\mathrm{N}_{11}-\mathrm{C}_{2}-\mathrm{C}_{4}-\mathrm{O}_{12}$ torsiehoeke van $-0.5(1)^{\circ}$ en $-1.0(1)^{\circ}$ versus $1.72(9)^{\circ}$. Dit is opmerklik dat die dihedriese hoek van een van die molekule in polimorf I $\left[31.67(6)^{\circ}\right]$ naby is aan dié van die molekuul in polimorf II $\left[31.97(4)^{\circ}\right]$ terwyl die ander baie groter op $58.99(4)^{\circ}$ is. Verbasend genoeg is 4-F-PhonyH die enigste verbinding wat 'n waterstof $\cdots$ halogeen interaksie vertoon, en ook in beide polimorwe.
Soos met 4-F-PhonyH vertoon die $P \overline{1}$ polimorwe van beide 4-Cl-PhonyH (IV) en 4-Br-PhonyH (VI) 'n groter 'byt' as hul ooreenstemmede $C_{c}$ polimorfiese vorme. Vir IV is die $\mathrm{N}_{11} \cdots \mathrm{O}_{12}$ afstand 2.705(2) $\AA$ en vir VI is dit 2.70(1) $\AA$, en dus 'n 0.029(4) $\AA$ tot 0.085(4) $\AA$ reeksverskil tussen IV en III, en

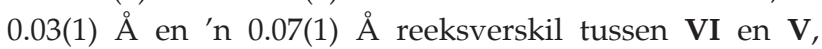
onderskeidelik. Verskille in intermolekulêre interaksies kan ook die rede wees vir die feit dat die polimorwe onderskeibaar is volgens kleur: die $C c$ polimorwe vir beide 4-Cl-PhonyH en 4-Br-PhonyH is geel terwyl die $P \overline{1}$ polimorwe kleurloos is.

Die eerste aanduiding van isomorfisme tussen 4-ClPhonyH en 4-Br-PhonyH is die vergelykbare eenheidselparameters, en al is vier onafhanklike molekule in die asimmetriese eenheid 'n skaars verskynsel, vertoon beide verbindings III en V hierdie eienskap. Isomorfisme is ook tussen verbindings IV en VI waargeneem. Die ruimtelike oorlegging van die twee stelle molekule vir verbindings III en $\mathbf{V}$, en IV en VI word in Figuur 4 vertoon.

Oor die algemeen vergelyk die bindingsparameters van die twee stelle molekule van die Cc polimorwe goed. Al kan verskille waargeneem word tussen molekules van dieselfde verbinding, verskyn hierdie verskille in beide die $\mathrm{Cl}$ en $\mathrm{Br}$ variante. Verskille word waargeneem tussen die geometriese parameters vir die verskeie molekules in elk van die $C_{c}$ polimorwe, maar hierdie verskille volg nie 'n neiging ten opsigte van molekuulposisie nie (m.a.w. vergelyking van molekule 1 en 3 , of 1 en 2 , of 2 en 3 ). Beide molekule 1 en 3 vertoon 'n positiewe dihedriese hoek, terwyl molekule 2 en 4 met 'n negatiewe dihedriese hoek georiënteer is. Ten spyte van die ooreenkomste in hierdie opsig verskil die dihedriese hoeke van molekule met dieselfde oriëntasies: $\left[13.4(1)^{\circ}\right.$ en $11.4(1)^{\circ}$ vir III, en $12.0(1)^{\circ}$ en $14.6(1)^{\circ}$ vir $\mathbf{V}$ vir molekule 1 en 3 , en 2 en 4 , onderskeidelik]. Die $\mathrm{N}_{11}-\mathrm{C}_{2}-\mathrm{C}_{3}-\mathrm{C}_{4}-\mathrm{O}_{12}$ gedeeltes van alle molekule is slegs effens vervorm soos bevestig deur die relatiewe klein $\mathrm{N}_{11}-\mathrm{C}_{2} \cdots \mathrm{C}_{4}-\mathrm{O}_{12}$ torsiehoeke [kleiner as $\left.3.4(3)^{\circ}\right]$. Beide verbindings bevat intermolekulêre interaksies tot so 'n mate dat hulle as kristallografiese polimere beskou kan word soos in Figuur 3 getoon. Die verskille tussen verbindings IV en VI kan binne eksperimentele fout as onbeduidend beskou word, maar die lae gehalte van die kristaldata vir VI moet hiermee in ag geneem word. Gevolglik word hierdie verbindings ter wille van volledigheid ingesluit, maar ' $n$ heropname moet onderneem word om die isomorwe ten volle te vergelyk.

\section{Samevatting}

Kennis van die fisiese eienskappe van ligandstelsels is belangrik vir toepassings in nywerheidsprosesse. Groot verskille is egter nie net tussen polimorwe van dieselfde tipe $4-\mathrm{X}$-PhonyH verbindings waargeneem nie, maar ook tussen 4-F-PhonyH en sy 4-Cl-PhonyH en 4-Br-PhonyH eweknieë. Vir beide weergawes van 4-Cl-PhonyH en 4-Br- 


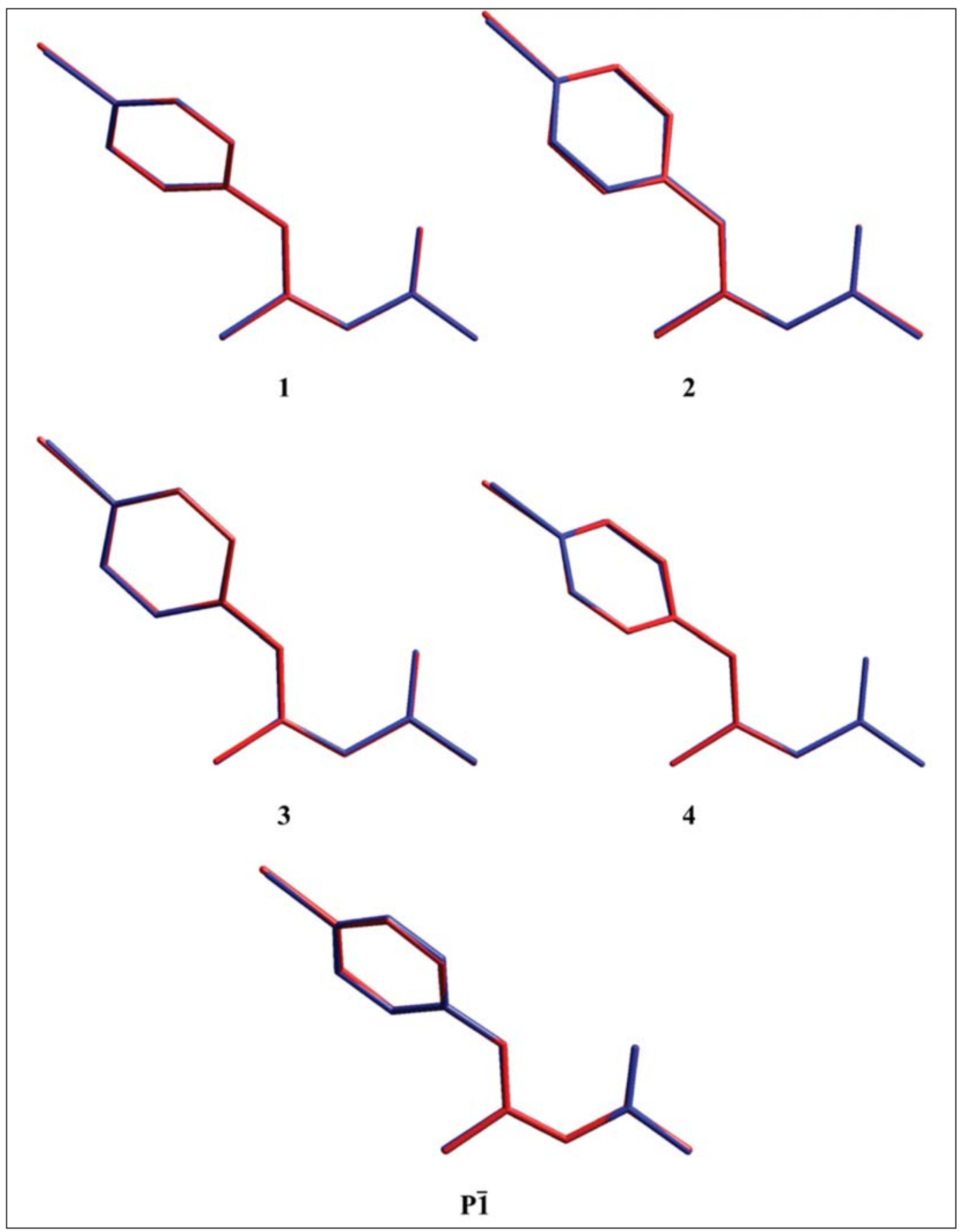

Figuur 4. Hyperchem ruimtelike oorleggings van die vier molekule van CC 4-Cl-PhonyH (III) met die vier ekwivalente molekule van Cc 4-Br-PhonyH (V), en $P \overline{1}$ 4-Cl-PhonyH (IV) met $P \overline{1}$ 4-Br-PhonyH (VI). 1: WGK $=0.0573 \AA$; 2 : WGK $=0.00855 \AA \AA$; $:$ WGK $=0.00542$

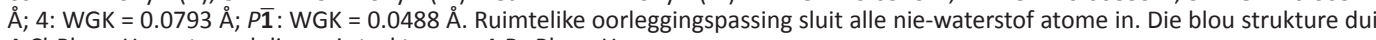
4-Cl-PhonyH aan terwyl die rooi strukture na 4-Br-PhonyH verwys.

PhonyH word isomorfisme tussen soortgelyke ruimtegroepe waargeneem. Polimorwe van 4-Cl-PhonyH en 4-BrPhonyH is duidelik deur middel van kleurverskille onderskeibaar.

Ten spyte van verskille in die elektroniese en steriese eienskappe van $\mathrm{F}, \mathrm{Cl}$, en Br blyk hierdie verskille nie 'n duidelike tendens te vorm in terme van die geometriese eienskappe van die vastetoestand verbindings van 4-X-PhonyH nie. Verdere studies is dus nodig waar hierdie verbindings as ligandstelsels implementeer word.

\section{Erkenning}

Ons spreek ons dank uit teenoor SASOL, die Suid-Afrikaanse Nasionale Navorsingstigting (SA-NNS/THRIP), en die Universiteit van die Vrystaat se Strategiese Akademiese Klusterinisiatief vir finansiële ondersteuning van hierdie projek. Deel van hierdie materiaal is gebaseer op werk wat deur die SA-NNS/THRIP onder toekenningsnommer GUN 2068915 ondersteun is. Menings, bevindings, gevolgtrekkings, of aanbevelings wat in hierdie artikel aangedui word, is dié van die outeurs en weerspieël nie noodwendig die standpunte van die SA-NRF nie. 


\section{Bydrae van elke outeur}

Die projek is deur GJSV konseptualiseer en uitgevoer, en die artikel geskryf en vertaal. ZGM het die sintese van sommige verbindings asook verfyning van sekere kristaldata behartig.

\section{Verwysings}

Bondi A. 1964. Van der Waals Volumes and Radii. The Journal of Physical Chemistry $68,441-451$.

Bruker SAINT-PLUS (including XPREP). Bruker AXS Inc., Madison, WI, 2004.

Bruker SADABS, Bruker AXS Inc., Madison, WI, 1998.

Chen H, Rhodes J. 1996. Schiff base forming drugs: mechanisms of immune potentiation and therapeutic potential. Journal of Molecular Medicine 74, 497-504.

Csöregh I, Brehmer T, Bombicz P, Weber E. 2001. Halogen …halogen versus $\mathrm{OH} \cdots \mathrm{O}$ supramolecular interactions in the crystal structures of a series of halogen
and methyl substituted cis-9,10-diphenyl-9,10-dihydroanthracene9,10-diols. Crystal Engineering 4, 343-357.

Da Silva MAVR, Da Silva MDMCR, Paiva JPA, Nogueira IMCS, Damas AM, Barkley JV, et al. 1993. Thermochemical and Crystallographic Studies of some p-Ketoimine Derivatives. Journal of the Chemical Society Perkin Transactions 2 1765-1769.

Fox DB, Liantonio R, Metrangolo P, Pilati T, Resnati G. 2004. Perfluorocarbonhydrocarbons

self-assembly: halogen bonding mediated intermolecular recognition. Journal of Fluorine Chemistry 125, 271-281.

Farrugia LJ. 2012. WinGX and ORTEP for Windows: an update. Journal of Applied Crystallography $45,849-854$.

Francl MM, Petro WJ, Hehre WJ, Binkley JS, Gordon MS, DeFree DJ, Pople JA. 1982. Self-consistent molecular orbital methods. XXIII. A polarization-type basis set for second-row elements. Journal of Chemistry and Physics 77, 3654-3665.

Gordon JC, Shukla P, Cowley AH, Jones JN, Keogh DW, Scott BL. 2002. Dialkyl aluminium amides: new reagents for the conversion of $\mathrm{C}=\mathrm{O}$ into $\mathrm{C}=\mathrm{NR}$ functionalities. Chemical Communications 2710-2711.

Groom CR, Bruno IJ, Lightfoot MP, Ward SC, 2016. The Cambridge Structural Database. Acta Crystallographica B72, 171-179.

HyperChem 7.5.2 release for Windows, Hypercube, Inc., Gainesville, Florida, USA.

Mantina M, Chamberlin AC, Valero R, Cramer CJ, Truhlar DG. 2009. Consistent van der Waals Radii for the Whole Main Group. The Journal of Physical Chemistry A 113, 5806-5812.
Nair VA, Suni MM, Sreekumar K. 2002. Catalytic effects of poly(methyl methacrylate)-supported $\beta$-diketone-linked palladium complexes in olefin oxidation. Proceedings of the Indian Academy of Sciences (Chemical Sciences) $114,481-486$.

Olejník R, Padělková Z, Horáček M, Ružička A. 2012. Structure of $\beta$-diketiminates and $\beta$-aminoketones made from anisidines or chloroanilines: tin and lithium complexes. Main Group Metal Chemistry 35, 13-27.

Parekh BB, Purohit DH, Sagayaraj P, Joshi HS, Joshi M. 2007. Growth and characterization of 4-(2-hydroxyphenylamino)-pent-3-en-2-one single crystals. Crystal Research and Technology 42, 407-415.

Putz H, Brandenburg K. 2018. Diamond - crystal and molecular structure visualization. Crystal Impact - GbR, Kreuzherrenstr 102, 53227 Bonn, Germany. http://www.crystalimpact.com/diamond

Pyżuk W, Krówczynsk A, Górecka E. 1993. Novel Series of Enaminoketone Liquid Crystals Having Hexatic Smectic B Phase. Molecular Crystals and Liquid Crystals 237, 75-84.

Sergienko VS, Garnovskii AD, Abramenko VL, Porai-Koshits MA. 1987 Synthesis and Crystal Structure of $\mathrm{N}$-(4-Bromophenyl)acetylacetoneimine. Koordinatsionnaya Khimiya (Russian Journal of Coordination Chemistry) 13, 1695-1709.

Shaheen F, Marchio L, Badshaha A, Khosac MK. 2006. (Z)-4-Anilinopent-3-en-2-one. Acta Crystallographica E62, 0873-0874.

Sheldrick GM. 2008. A short history of SHELX. Acta Crystallographica A64, 112-122.

Tan HY, Loke WK, Tan YT, Nguyen N-T. 2008. A lab-on-a-chip for detection of nerve agent sarin in blood. Lab Chip 8, 885-891.

Venter GJS, Steyl G, Roodt A. 2010a. 4-(2-Methylanilino)pent-3-en-2-one. Acta Crystallographica E66, 01593-01594.

Venter GJS, Steyl G, Roodt A. 2010b. 4-[(4-Methylphenyl)amino]pent-3-en-2-one. Acta Crystallographica E66, 03011-03012.

Venter GJS, Steyl G, Roodt A. 2011. 4-(2,6-Dibromo-4-fl uoroanilino)pent-3-en-2one. Acta Crystallographica E67, 03092-03093.

Venter GJS, Steyl G, Roodt A. 2012a. 4-(2,3-Dimethylanilino)pent-3-en-2-one. Acta Crystallographica E68, 02930-02931.

Venter GJS, Steyl G, Roodt A. 2012b. 4-(2-Chlorophenylamino)-pent-3-en-2-one. Acta Crystallographica E68, 03101-03102.

Venter GJS, Steyl G, Roodt A. 2013. 2,6-Dichloroaniline-4-(2,6-dichloroanilino) pent-3-en-2-one (1/2). Acta Crystallographica E69, 034-035.

Venter GJS, Steyl G, Roodt A. 2014. Solid state and theoretical study of structural properties induced by step-wise chloro functionalization in dicarbonyl[2-(phenylamino)pent-3-en-4-onatolrhodium(I) complexes. Journal of Coordination Chemistry 67, 176-193.

Xia M, Wu B, Xiang G. 2008. Synthesis, structure and spectral study of two types of novel fluorescent BF2 complexes with heterocyclic 1,3-enaminoketone ligands. Journal of Fluorine Chemistry 129, 402-4 08. 


\section{Ondersteunende Materiaal}

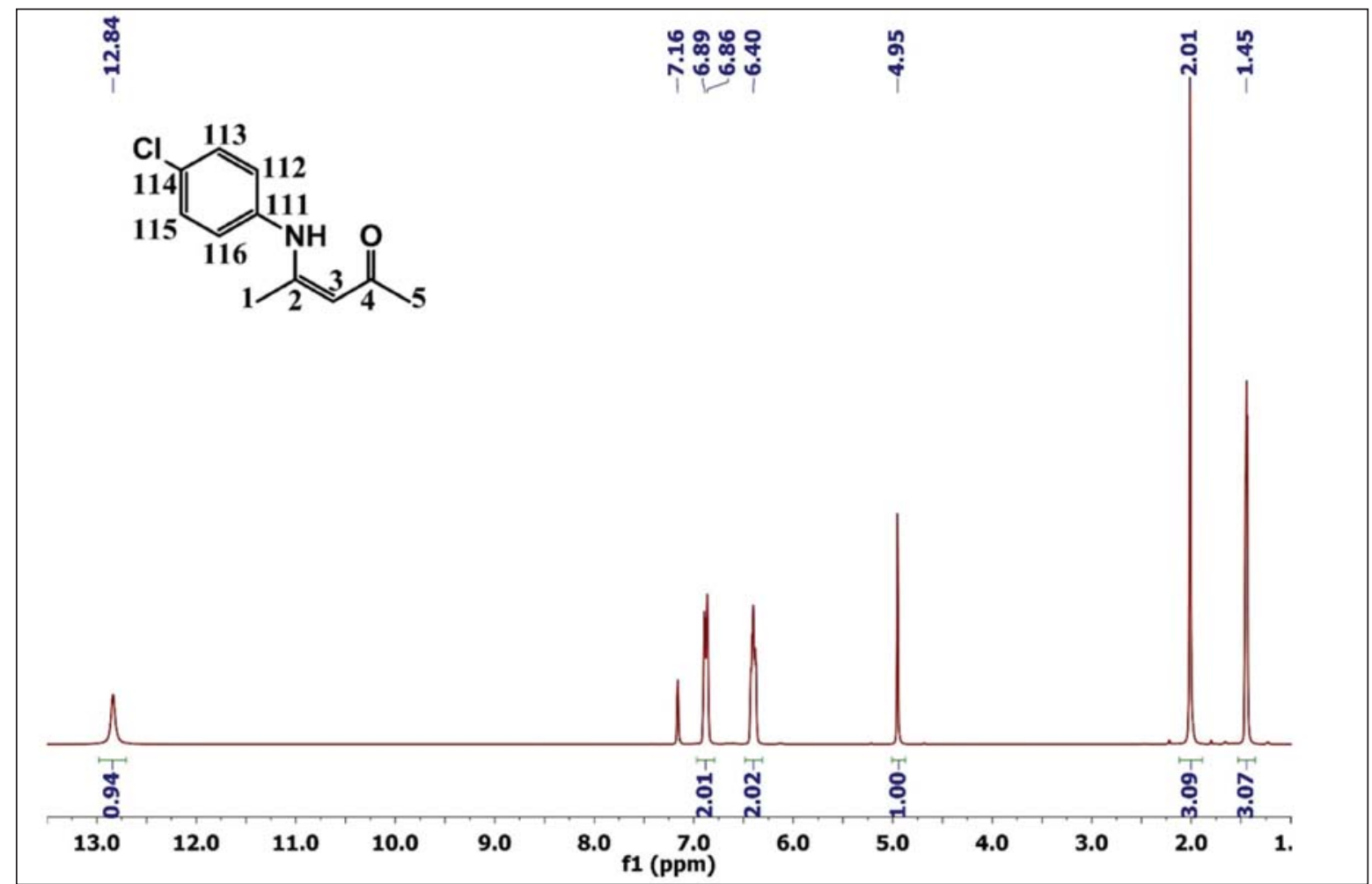

FIGUUR A.1: ${ }^{1} \mathrm{H}(300.13 \mathrm{MHz}) \mathrm{KMR}$ spektrum van $4-\mathrm{Cl}-\mathrm{PhonyH}$ in $\mathrm{C}_{6} \mathrm{D}_{6}$ by $25^{\circ} \mathrm{C}$.

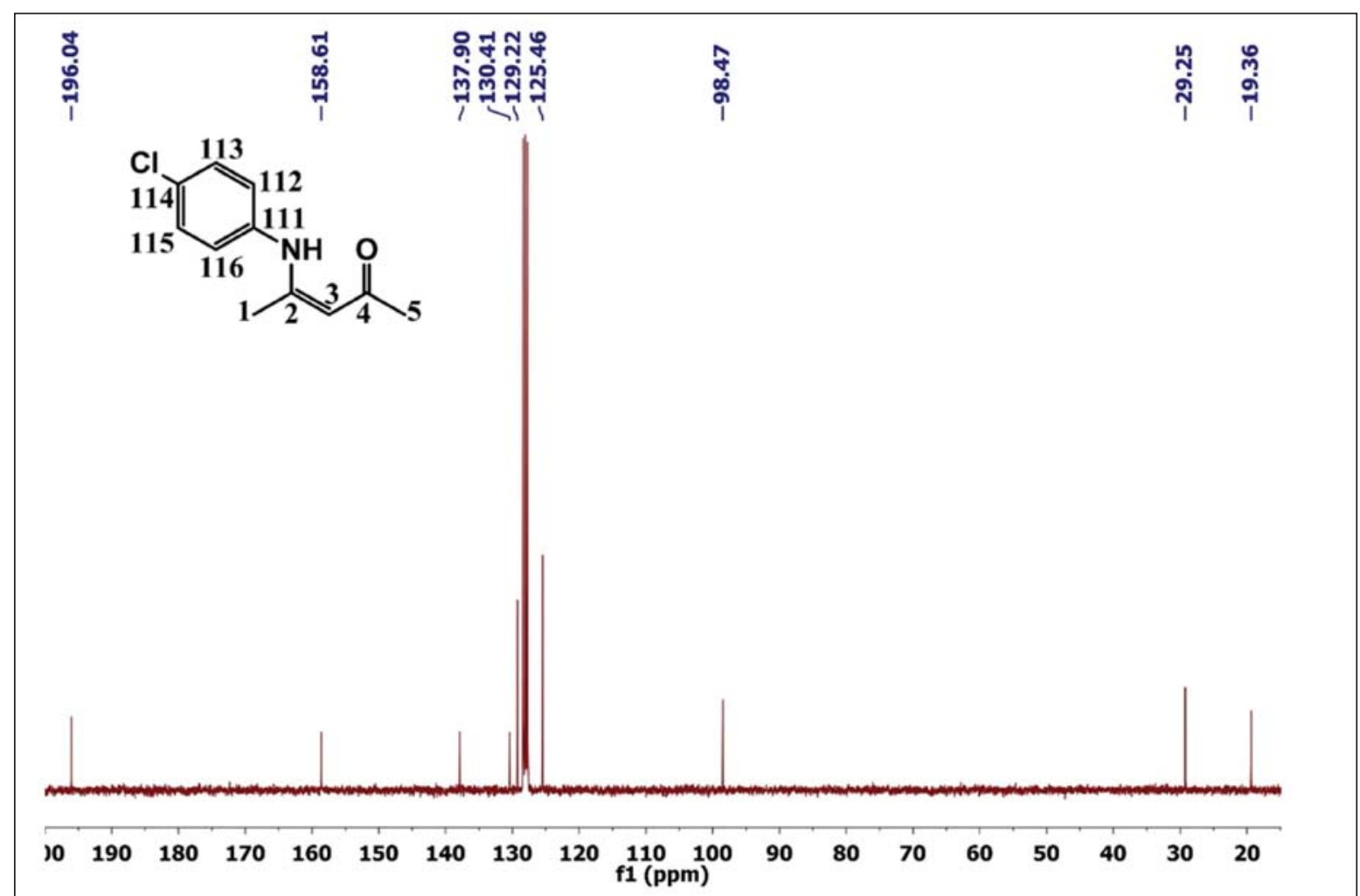

FIGUUR A.2: ${ }^{13} \mathrm{C}(75.48 \mathrm{MHz})$ KMR spektrum van 4-Cl-PhonyH in $\mathrm{C}_{6} \mathrm{D}_{6}$ by $25^{\circ} \mathrm{C}$. 
TABEL 1: Strukturele inligting van die halogeenderivate van PhonyH.

\begin{tabular}{|c|c|c|c|c|c|c|}
\hline Verbinding & $D-H \cdots A$ & $d_{D-H}(\AA)$ & $d_{H \cdots A}(\AA)$ & $d_{D \cdots A}(\AA)$ & $<_{\mathrm{DHA}}\left({ }^{\circ}\right)$ & Simmetriekode \\
\hline \multirow[t]{7}{*}{ I } & $\mathrm{N}_{11}-\mathrm{H}_{11} \cdots \mathrm{O}_{12}$ & $0.86(2)$ & $1.98(2)$ & $2.668(1)$ & $136(2)$ & \\
\hline & $\mathrm{N}_{21}-\mathrm{H}_{21} \cdots \mathrm{O}_{22}$ & $0.89(2)$ & $2.01(2)$ & $2.709(2)$ & $134(2)$ & \\
\hline & $\mathrm{N}_{11}-\mathrm{H}_{11} \cdots \mathrm{O}_{22}$ & $0.86(2)$ & $2.31(2)$ & $2.971(2)$ & $133(2)$ & \\
\hline & $\mathrm{N}_{21}-\mathrm{H}_{21} \cdots \mathrm{O}_{12}$ & $0.89(2)$ & $2.33(2)$ & $3.041(2)$ & $137(1)$ & \\
\hline & $\mathrm{C}_{6}-\mathrm{H}_{6 \mathrm{~A}} \cdots \mathrm{F}_{24}{ }^{\mathrm{i}}$ & 0.98 & 2.5 & $3.420(2)$ & 156.8 & $2-x, 1-y, 1-z$ \\
\hline & $\mathrm{C}_{113}-\mathrm{H}_{113} \cdots \mathrm{O}_{12}{ }^{i i}$ & 0.95 & 2.54 & $3.349(2)$ & 142.8 & $1-x, 2-y, 2-z$ \\
\hline & $\mathrm{C}_{213}-\mathrm{H}_{213} \cdots \mathrm{O}_{22}$ iii & 0.95 & 2.43 & $3.273(1)$ & 148.1 & $1-x, 1-y, 1-z$ \\
\hline \multirow[t]{4}{*}{ II } & $\mathrm{N}_{11}-\mathrm{H}_{11} \cdots \mathrm{O}_{12}$ & $0.88(2)$ & $1.89(2)$ & $2.641(2)$ & $142(2)$ & \\
\hline & $\mathrm{C}_{1}-\mathrm{H}_{1 \mathrm{~A}} \cdots \mathrm{F}_{14}^{\mathrm{iv}}$ & 0.98 & 2.48 & $3.326(2)$ & 144.9 & $2-x, 1 / 2+y, 1 / 2-z$ \\
\hline & $\mathrm{C}_{1}-\mathrm{H}_{1 \mathrm{~B}} \cdots \mathrm{O}_{12}^{\mathrm{v}}$ & 0.98 & 2.63 & $3.534(1)$ & 143.6 & $x, 11 / 2-y,-1 / 2+z$ \\
\hline & $\mathrm{C}_{116}-\mathrm{H}_{116} \cdots \mathrm{O}_{12}^{\mathrm{vi}}$ & 0.95 & 2.53 & $3.407(2)$ & 153.6 & $1-x, 1-y, 1-z$ \\
\hline \multirow[t]{9}{*}{ III } & $\mathrm{N}_{11}-\mathrm{H} 11 \cdots \mathrm{O}_{12}$ & $0.81(2)$ & $2.02(3)$ & $2.673(3)$ & $138(2)$ & \\
\hline & $\mathrm{N}_{21}-\mathrm{H}_{12} \cdots \mathrm{O}_{22}$ & $0.83(2)$ & $1.92(3)$ & $2.624(2)$ & $142(2)$ & \\
\hline & $\mathrm{N}_{31}-\mathrm{H}_{31} \cdots \mathrm{O}_{32}$ & $0.89(3)$ & $1.89(3)$ & $2.631(2)$ & $140(2)$ & \\
\hline & $\mathrm{N}_{41}-\mathrm{H}_{41} \cdots \mathrm{O}_{42}$ & $0.89(3)$ & $1.99(3)$ & $2.682(2)$ & $134(2)$ & \\
\hline & $\mathrm{C}_{6}-\mathrm{H}_{6 \mathrm{C}} \cdots \mathrm{O}_{12}$ & 0.98 & 2.51 & $3.341(3)$ & 142 & \\
\hline & $\mathrm{C}_{11}-\mathrm{H}_{11 \mathrm{~B}} \cdots \mathrm{O}_{42}$ & 0.98 & 2.42 & $3.266(3)$ & 143.9 & \\
\hline & $\mathrm{C}_{12}-\mathrm{H}_{312} \cdots \mathrm{O}_{22}$ & 0.95 & 2.54 & $3.287(3)$ & 135.2 & \\
\hline & $\mathrm{N}_{11}-\mathrm{H}_{11} \cdots \mathrm{O}_{42}$ vii & $0.81(2)$ & $2.49(2)$ & $3.136(2)$ & $138(2)$ & $-1 / 2+x,-1 / 2+y, z$ \\
\hline & $\mathrm{N}_{41}-\mathrm{H} 4_{1} \cdots \mathrm{O}_{12}^{\text {viii }}$ & $0.89(3)$ & $2.44(3)$ & $3.166(2)$ & $139(2)$ & $1 / 2+x, 1 / 2+y, z$ \\
\hline \multirow[t]{2}{*}{ IV } & $\mathrm{N}_{11}-\mathrm{H}_{11} \cdots \mathrm{O}_{12}$ & $0.87(3)$ & $2.00(2)$ & $2.705(2)$ & $137(2)$ & \\
\hline & $\mathrm{N}_{11}-\mathrm{H} 11 \cdots \mathrm{O}_{12}{ }^{\mathrm{ix}}$ & $0.87(3)$ & $2.48(3)$ & $3.316(2)$ & $130(2)$ & $-x, 2-y, 1-z$ \\
\hline \multirow[t]{11}{*}{ V } & $\mathrm{N}_{11}-\mathrm{H}_{11} \cdots \mathrm{O}_{12}$ & $0.886(3)$ & $2.003(2)$ & $2.675(4)$ & 131.50(18) & \\
\hline & $\mathrm{N}_{21}-\mathrm{H}_{21} \cdots \mathrm{O}_{22}$ & $0.899(3)$ & $1.802(2)$ & $2.635(3)$ & 152.98(19) & \\
\hline & $\mathrm{N}_{31}-\mathrm{H}_{31} \cdots \mathrm{O}_{32}$ & $0.906(3)$ & $1.832(2)$ & $2.629(3)$ & $145.5(2)$ & \\
\hline & $\mathrm{N}_{41}-\mathrm{H}_{41} \cdots \mathrm{O}_{42}$ & $0.840(3)$ & $1.972(2)$ & $2.665(4)$ & 139.24(19) & \\
\hline & $\mathrm{C}_{6}-\mathrm{H}_{6 \mathrm{~B}} \cdots \mathrm{O}_{12}$ & 0.98 & 2.43 & $3.291(4)$ & 146.2 & \\
\hline & $\mathrm{C}_{11}-\mathrm{H}_{11 \mathrm{~B}} \cdots \mathrm{O}_{42}$ & 0.98 & 2.53 & $3.325(4)$ & 138.6 & \\
\hline & $\mathrm{C}_{216}-\mathrm{H}_{216} \cdots \mathrm{O}_{32}$ & 0.95 & 2.52 & $3.312(4)$ & 141.1 & \\
\hline & $\mathrm{C}_{312}-\mathrm{H}_{312} \cdots \mathrm{O}_{22}$ & 0.95 & 2.61 & $3.396(4)$ & 139.7 & \\
\hline & $\mathrm{N}_{11}-\mathrm{H}_{11} \cdots \mathrm{O}_{42}{ }^{\mathrm{x}}$ & $0.886(3)$ & $2.473(2)$ & $3.217(4)$ & $141.92(18)$ & $-1 / 2+x, 1 / 2+y, z$ \\
\hline & $\mathrm{N}_{41}-\mathrm{H}_{41} \cdots \mathrm{O}_{12}{ }^{\mathrm{xi}}$ & $0.840(3)$ & $2.527(2)$ & $3.180(4)$ & 135.41(19) & $1 / 2+x,-1 / 2+y, z$ \\
\hline & $\mathrm{C}_{16}-\mathrm{H}_{16} \mathrm{~B} \cdots \mathrm{O}_{32}{ }^{\mathrm{xii}}$ & 0.98 & 2.59 & $3.555(4)$ & 166.5 & $-1 / 2+x,-1 / 2+y, z$ \\
\hline \multirow[t]{3}{*}{ VI } & $\mathrm{N}_{11}-\mathrm{H}_{11} \cdots \mathrm{O}_{12}$ & $0.97(8)$ & $1.89(8)$ & $2.70(1)$ & 139(6) & \\
\hline & $\mathrm{N}_{11}-\mathrm{H}_{11} \cdots \mathrm{O}_{12} \mathrm{xii}$ & $0.97(8)$ & $2.46(7)$ & $3.16(1)$ & $129(5)$ & $1-x, 1-y, 1-z$ \\
\hline & $\mathrm{N}_{11}-\mathrm{H}_{11} \cdots \mathrm{O}_{12}{ }^{\text {xiv }}$ & $1.07(7)$ & $2.44(7)$ & $3.35(1)$ & $141(6)$ & $1+x, y, z$ \\
\hline
\end{tabular}

